\title{
Clinical Diagnosis of Bone Tumor and X-ray Application for Patients with Bone Tumor
}

\author{
Rui Jiang ${ }^{1}$, Ziyan Zhang ${ }^{2}$, Han $\mathrm{Wu}^{1}$ and Chao $\mathrm{Zhang}^{3}$ \\ ${ }^{1}$ Department of orthopedics, China-Japan Union Hospital of Jilin University, Jilin 130033, China \\ ${ }^{2}$ Department of orthopedics, The Second Hospital of Jilin University, Jilin 130041, China \\ ${ }^{3}$ Department of ophthalmology, The Second Hospital of Jilin University, Jilin 130041, China \\ Corresponding Author: Chao Zhang: zhangchao0701@163.com
}

Keywords: X-ray; bone tumor; diagnosis

\begin{abstract}
Objective: To analyze the application value of X-ray in the diagnosis of bone tumor for patients. Methods: With the retroactive analysis on the clinical data of 52 patients with bone tumor, which were accepted by the hospital and received X-ray examination from January 2013 to January 2014, the rate of accurate diagnosis through X-ray was calculated. Results: 25 out of 52 patients manifested benign bone tumor, accounting for $48.08 \%$, while 27 patients were diagnosed to have malignant bone tumor, accounting for 51.92\%. The rate of accurate diagnosis through X-ray was 88.46\% (46/52). Conclusion: X-ray had very high value of application in the diagnosis of bone tumor for patients, and could provide reference for clinical diagnosis and therapy of bone tumor, so it was worth promotion.

Orthopaedic surgeons often ask via telephone or online about what they should do when the postoperative pathological conditions are not as expected before operation after treating the bone and soft tissue tumors, or there are some unknown tumors. In the outpatient department of bone tumor, patients are often asked by local surgeons to seek a specialized hospital as soon as possible for better treatment scheme even before their stitches from operation are removed. As a surgeon specialized in bone tumor, the author firmly believes that we could provide better services for patients and avoid improper therapy if we attach importance to the standardization of clinical therapy and diagnosis of bone tumor, gather the complete images and data at the beginning of diagnosis and therapy, and utilize the histopathology for prudent diagnosis, identification and diagnosis. Bone tumor is a very rare tumor of kinetic system. The primary malignant bone tumors are represented by osteosarcoma, Ewing's sarcoma, and malignant fibrous histiocytoma, while benign bone tumors mainly include bone cyst, aneurysmal bone cyst, fibrous dysplasia of bone and other tumor-like diseases as well as giant cell tumor of bone. Among senior people, cancer with bone metastasis is the commonest disease. The primary malignant bone tumor, which often happens to teenagers and young adults, exists around the knee joint, so it severely affects the motor functions of patients. At the time of preliminary diagnosis and therapy, $80 \%$ of the patients have potential lung metastasis, and the long-term survival rate is lower than $20 \%$ if they receive the surgical treatment only. Before the 1970s, surgical treatment mainly relied on amputation, which caused the physical disability and functional disorders of patients. Along with the improved understanding about the biological behaviors of tumor, the progress of medical imaging technology, the soaring development of surgical techniques, and especially the application of chemotherapy in malignant tumors, all kinds of techniques for limb salvage had been constantly and successfully practiced to restore the functions of extremities for many patients, and the five-year survival rate was improved steadily to $70 \%$. In the past, tumor treatment relied on the surgical removal of lesions, but the treatment method has been significantly changed today. Simple bone cyst happens to the metaphysic of children, and may reoccur and affect the development of extremities if it is not properly treated. In today's treatment, several intracapsular penetrations with needles are performed to scraping off the capsular walls for lavage, and methylprednisolone is injected to facilitate the self-healing of bone cyst.
\end{abstract}


Palliative care was practiced for cancer with bone metastasis in the past. Today, active surgical interference is performed, and all kinds of surgical techniques are widely applied, in order to improve the quality of life for patients. However, orthopaedic surgeons should realize that orthopaedic surgery is only a part of integrated treatment, so the quality of life can be improved for patients only through preventing bone fracture and stabilizing spine. In the past twenty years, the professional knowledge about bone tumor has been greatly increased thanks to the contributions from senior experts and the continuous efforts of junior experts. Before the 1990s, senior experts took a lead in the clinical and laboratory studies on bone tumor, such as Professor Song Xianwen of Beijing Jishuitan Hospital, Professor Feng Chuanhan of Peking University People's Hospital and Professor Huang Chengda of The First Affiliated Hospital, Sun Yat-sen University, who advocated the idea of diagnosis by combining the clinical, imaging and pathological data. Professor Song Xianwen put forth the method of tumor-affected bone resection and 95\% alcohol inactivated tumor-affected bone replantation for the first time, which helped a lot of patients with bone tumor salvage their limbs and restore the functions of joints. The method has been widely practiced in China. In the past, only Beijing Jishuitan Hospital established the department of bone tumor in China, but more than ten hospitals have organized their independent department of bone and soft tissue treatment today, and the treatment of bone and soft tissue tumor is available in more than fifty hospitals throughout the country. The progress of medical science and technology brings the hope of cure to patients and their families, while the rapid economic development enables more and more patients with bone and soft tissue tumors to seek treatments. As the diagnosis and therapy of cancers in other departments is improved to make patients live longer, more and more patients suffer from cancer with bone metastasis. Due to various factors, the number of patients with bone and soft tissue tumors was doubled and redoubled. These patients go to the department of bone tumor for treatment, but most of them are preliminarily diagnosed in general orthopaedics or oncological surgery. Meanwhile, surgeons, who acquire all kinds of advanced surgical techniques, also desire to improve the treatment level of bone tumor and provide services for patients. Moreover, general hospitals are required to conduct the highly challenging diagnosis and therapy of bone tumors, so as to become Grade III hospitals. What is more important, the orthopaedic surgeons in grassroots hospitals and large general hospitals lack the special training about bone and soft tissue tumors, so the number of patients improperly treated in the bone and soft tissue tumor diagnosis and therapy centers throughout the country is increasing noticeably. Due to the current clinical conditions, we should attach importance to the standardization of clinical diagnosis and therapy for bone tumor, so popularizing the guidelines for diagnosis and therapy is of great significance. If these guidelines are ignored, severe hazards may be caused. If patients receive nonstandard diagnosis and therapy, they should be often retreated as the postoperative pathological diagnosis does not meet the requirements of surgical treatment. Moreover, improper treatment may be practiced due to the defective diagnosis pathway, which may eventually cause the loss of extremities and even cost the life of patients.

Defective diagnosis pathway means that it is difficult to determine the scope of reoperation due to too simple preoperative examination, lack of complete radiograph, enhanced CT, MRI and ECT, etc., and no preoperative examination and diagnosis based on histopathology. Bone tumor diagnosis and therapy center should assume the responsibilities, voluntarily pay attention to the guidelines for diagnosis and therapy of bone tumor, exert their strong influence and demonstration effect in the academic communication, and promote the guidelines for clinical diagnosis and therapy of bone tumor at different levels throughout the country for a long time. The bone and soft tissue tumor diagnosis and therapy centers throughout the country are obligated to promote the guidelines for diagnosis and therapy for surgeons of general orthopaedics, while the surgeons of general orthopaedics should also receive the continued education regarding oncologic knowledge, so patients will benefit from the precious medical development and progress. Meanwhile, surgeons should constantly gather and accumulate the consensuses on the clinical diagnosis and therapy of bone tumor, improve the guidelines, and enhance the quality of scientific research with cooperation among multiple centers. The bone tumor specialty is an inter-discipline of oncology, orthopaedics and osteopathic internal medicine, so surgeons should not attach importance only to the 
reconstruction of bone defects, but also pay more attention to the cure of bone tumor. According to evidence-based medicine, if surgeons do not fully consider the oncologic characteristics of bone, any successful surgery may lead to the failure of treatment, which will cause the loss of extremities and even cost the life of patients. Hence, we should attach more importance to the standardization of clinical diagnosis and therapy pathway and the integrated treatment of bone tumor. Under this circumstance, CSCO Expert Committee for Osteosarcoma was incorporated with the focus on single disease in 2009, and dedicated to gain the consensuses from experts through their discussions on the clinical and treatment pathway of bone tumor. In Jiangsu, Zhejiang, Hebei, Shijiazhuang, Beijing and Tianjin, it has organized many expert discussions for many times. Also, it plans to release the Guideline for Clinical Diagnosis and Therapy of Conventional Osteosarcoma. Along with the continuous development of bone tumor therapy, clinical medical research has been carried out in a more and more prudent manner. Hence, the experts with achievements should offer the education regarding standardization on the platform at multiple levels, and engage in the cooperation with multiple centers to implement the unified therapy pattern, which is of great significance to improving the professional level of bone tumor diagnosis and therapy.

Most bone tumors happen due to heredity and environment. If they are not treated in a timely and effective manner, these tumors may threaten the life of patients [1-2]. Accurate clinical diagnosis provides a guarantee for treatment effectiveness and improved prognosis of patients with bone tumors. As many types of bone tumors come from complicated sources and have much different manifestations of medical imaging, the treatment of bone tumors is adversely affected. At present, X-ray is the commonest method for the diagnosis of bone tumors. As it can vividly display the features of diseased tissues, X-ray is an ideal way of examination. This paper presents a retrospective analysis on the clinical data of patients with bone tumors, and explores the application value of this examination method, with an aim to provide reference for the diagnosis of this disease.

\section{Data and Methods}

1.1 General Data. From the patients with bone tumors received and treated by the hospital from January 2013 to January 2014, 52 cases were selected, and these patients were all definitely diagnosed through pathological examination, and did not have any severe functional disorders of

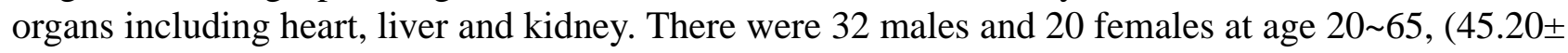
7.35) years old on average. In terms of tumor position, there were 10 cases at tibia, 2 cases at spine, 14 cases at humerus, 18 cases at femur and 8 cases at ribs.

1.2 Diagnostics. All patients received the X-ray examination, which was carried out strictly in accordance with the relevant standards. During the examination, regular front and lateral radiography was performed. The results of X-ray examination and pathological examination were comparatively analyzed.

1.3 Statistical Analysis. All data were analyzed in the software SPSS 15.0. Quantitative data were described by mean \pm standard deviation $(\mathrm{x} \pm \mathrm{s})$, while the $\mathrm{t}$ test was performed to compare the difference between groups. The group comparison for qualitative data relied on the $\chi 2$ test. When $\mathrm{P}<0.05$, the difference is statistically meaningful.

\section{Results}

2.1 Results of Pathological Examination. As revealed in the results of pathological examination, there were 21 benign bone tumors, accounting for $40.38 \%$, mainly including giant cell tumor of bone ( 9 cases) and osteochondroma (12 cases); and 31 malignant bone tumors, accounting for 59.62\%, mainly including chondrosarcoma (10 cases), osteosarcoma (15 cases), lymphosarcoma (4 cases) and chordoma (2 cases).

2.2 Results of X-ray Examination. Among 52 patients, there were 25 cases with the manifestation of benign bone tumors, accounting for 48.08\%; and 27 cases with the manifestation of malignant bone tumors, accounting for $51.92 \%$. The rate of accurate diagnosis with X-ray was $88.46 \%(46 / 52)$. 
2.3 Features of X-ray Imaging. (1)X-ray features of benign bone tumors: Most patients had intact bone shell, clear tumor contour and no periosteal reaction. A few patients showed periosteal reaction, but their periosteum was still intact, while some soft tissue masses were visible and their edges were smooth and had no change of infiltration. (2) X-ray features of malignant bone tumors: Most patients experienced the disappearance of bone cortex, destruction of bone and poor clarity, while a few soft tissue masses were visible in some cases of osteosarcoma. A few patients experienced local capsular swelling, whose edges were clear. Along with the extension of course, the edges of infiltration changed.

\section{Discussions}

In recent years, the increasing incidence of tumors is accompanied by the increasing number of patients with bone tumors, which causes much attention and concern of clinical practitioners [3]. Bone tumors are classified into benign and malignant tumors. Among them, benign bone tumors cause less pain, and are easily rooted with good prognosis, while malignant bone tumors spread rapidly and cause worse pain along with the extension of course, and may experience the metastasis of lesions. If occurred, malignant bone tumors would severely affect the physical health of patients and even threaten their life. For this reason, timely and accurate diagnosis for these patients in their early stage can improve the effectiveness of treatment and extend the life of patients. Along with the continuous improvement of medical level, and the increasing technical level of medical imaging, medical imaging examination will be performed to help clinical practitioners accurately identify the position, pathological nature and clinical classification of bone tumor. At present, the common techniques for medical imaging include CT, MRI, ultrasonic, and X-ray. Among them, X-ray examination is the common and most important method for examination of bone tumors. In this examination, the three-dimensional structure of human body is compressed into a plane image to help clinical surgeons check the organizational structure of human body conveniently, and accurately identify the pathological range, position and type of bone tumor. On the other hand, $\mathrm{X}$-ray examination can also reflect the relationship between tumor and its peripheral tissues, so it can offer great guidance for clinical surgeons to judge whether bone tumor is benign or malignant and there is metastasis [4]. Meanwhile, X-ray examination features simple operation, a great amount of information, and low cost, so it is easily accepted by patients, and has gradually become the first choice in the diagnosis of bone tumor. Nevertheless, the occurrence of bone tumor relates closely to heredity and other factors, so clinical surgeons should learn about the medical history and family medical history of patients as much as possible in the practice. If it is a benign tumor, surgeon should ask patient whether he experienced any trauma before, make a judgment based on the results of other examinations and the clinical symptoms of patient if necessary, and confirm the size and source of tumor. If it is suspected to be malignant, surgeon should first find out whether the patient has any history of tumor, and then identify the type of tumor, e.g. myeloma and spinal tumor. Additionally, the medical imaging manifestation of bone tumors is very complicated, and different types of tumors have much different manifestations in X-ray examination, which causes some difficulties in the clinical diagnosis of bone tumors. As shown in the study, X-ray examination identified 25 cases of benign bone tumor and 27 cases of malignant bone tumors among 52 patients, so its rate of accurate diagnosis was $88.46 \%$ after comparison with the results of pathological examination. On the basis of the above analysis, clinical surgeons should combine X-ray with other techniques for integrated judgment to improve the rate of accurate diagnosis for bone tumors. For instance, to identify whether a bone tumor is benign or malignant, it is necessary to consider the possible malignant change of tumor on the basis of X-ray features if there are noticeable soft tissue masses projecting out of the bone with benign bone tumor, and bony shell is entirely invaded by tumor. Differently, malignant bone tumors are mainly reflected in periosteal reaction and various shapes, and may penetrate periosteum when the patient's conditions get worse. Above all, X-ray has very high application value in the diagnosis of bone tumor for patients, so it is meaningful to promote it clinically. 


\section{Acknowledgments}

This work was supported by The study of c-Myc and mTOR inhibitors in the combined targeting therapy of osteosarcoma.

\section{References}

[1] Wu Hongying, Liang Huimin, Zeng Xiangjie. Application of CT window technology in diagnosis of malignant bone tumors [J]. Journal of Clinical Radiology, 2001, 20 (2): 132-133.

[2] Yu Xiuchun, Liu Xiaoping, Zhou Yin et al. Complementary effect of CT and X-ray examination in diagnosis of bone tumors [J]. Orthopedic Journal of China, 1998,5(3):265.

[3] Ding Chenglong, Liu Aihua, Wang Guangjun. The CT manifestation of the osteosarcoma [J]. Journal of China Clinic Medical Imaging, 2000, 11(4):291-292.

[4] Cao Laibin, Xu Aide, Xu Deyong. Practical Bone and Joint Imaging Diagnosis [M]. Ji’nan: Shandong Science and Technology Press, 1998. 326-338. 